Part of Journal of Research of the National Bureau of Standards, Volume 17, September 1936

\title{
IONIZATION OF AIR BY LENARD RAYS
}

\author{
By Lauriston S. Taylor
}

\section{ABSTRACT}

Lenard rays in atmospheric air have been measured by means of simple ionization chambers. At ordinary intensities, encountered with Lenard rays, it is impossible to saturate such ionization chambers and this has resulted in neglect of the method for practical purposes. It has been found, however, that plotting the reciprocal current against the reciprocal voltage, a straight line results, which can be extrapolated to $1 / V=0$; thereby giving the current at infinite field. A number of such cases are given. Unipolar conductivity is evident in at least one ionization chamber.

\section{CONTENTS}

I. Introduction

Page

1. Introduction

II. Experimental arrangement._.

III. Experimental results _. _.

1. Current-voltage curves 2. Reciprocal current-voltage curves... 486

IV. Discussion

\section{INTRODUCTION}

In 1930 a study was undertaken to arrive at a reliable method for measuring Lenard rays in such applications as the activation of ergosterol and the study of cathode ray biological effects. ${ }^{1}$

By intensity of Lenard rays is meant the rate of passage of electrons across unit area normal to the beam. ${ }^{2}$ This does not involve the velocity distribution of the electrons within the beam. After investigating several, it was decided that the most direct method of measuring Lenard ray intensities was probably that employing a faraday collector. ${ }^{3}$

On the other hand, the measurement of Lenard rays with an ionization chamber involves problems analogous to those commonly encountered in the measurement of X-rays and has the important advantage of being more simply related to the absorption in biological media. The ionization current is proportional to the fractional part of the energy of the whole beam, which is absorbed in the measuring volume of the chamber. This in turn depends upon the quality or velocity distribution of the electrons - a quantity which, at best, can only be expressed in terms of some average distribution or composite absorption coefficient. ${ }^{4}$

1 This work was discontinued in 1932, and since the apparatus has largely been disassembled, it is not feasible at present to investigate further some of the problems suggested by a study of the old data.

2 L. S. Taylor, Radiology 12, 294 (1929).

3 L. S. Taylor, BS J. Research 7, 57 (1931) RP332

L. S. Taylor, BS J. Research 12, 401 (1934) RP636; Radiology 22, 445 (1934). 
In addition, the air ionization method of measurement offers a convenient mode of control and is presumably proportional to the intensity of a given quality. The use of a simple ionization chamber was tried, but it was abandoned because of inability to reach saturation without causing a disruptive breakdown of the air by collision ionization under the high electrostatic fields employed.

Recent studies ${ }^{5}$ have led to an application of Jaffe's theory of columnar ionization, ${ }^{6}$ whereby it is possible to derive the saturation current value in liquids exposed to X-rays. Clay and Van Tijn ${ }^{7}$ and Zanstra ${ }^{8}$ have independently arrived at similar results in the case of high-pressure gaseous ionization chambers exposed to gamma
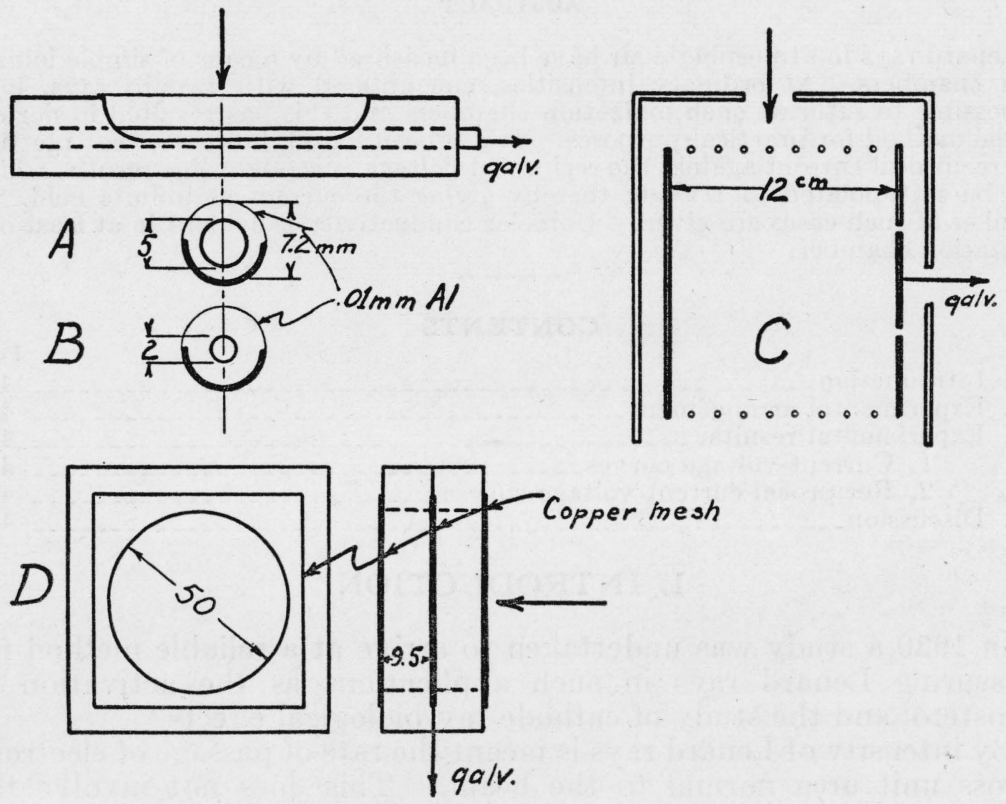

FIgURe 1.-Ionization chambers used with Lenard rays.

rays. These studies have led us to investigate the shape of the saturation curves produced by Lenard rays in air at normal pressure.

\section{EXPERIMENTAL ARRANGEMENT}

The detailed description of the apparatus has been given in an earlier paper. ${ }^{9}$ The cathode rays were produced in a sealed-off Lenard tube with a thin glass window, designed by Slack ${ }^{10}$ and operated from a high-voltage source of the Villard rectifier type. The voltage was approximately a sine wave above ground with a peak value of $150 \mathrm{kv}$. Input voltage was supplied by a synchronous motor-generator set and hence reasonably steady. Output voltage was controlled and measured directly with a high-resistance voltmeter. Tube current, measured between anode and ground, was maintained at $21 \times 10^{-6} \mathrm{amp}$.

s F. L. Mohler and L. S. Taylor, BS J. Research 13, 659 (1934) RP733.

6 G. Jaffe, Ann. Phys. 42, 303 (1913).

7 J. Clay and M. A. Van Tijn, Physica 2, 825 (1935).

8 H. Zanstra, Physica 2, 817 (1935)

- L. S. Taylor, BS J. Research 7, 57 (1931) RP332.

10 C. M. Slack, J. Opt. Soc. Am. 18, 123 (1929). 
The ionization chamber was mounted on a track in front of the tube window. The ionization current $\left(10^{-7}\right.$ to $\left.10^{-8} \mathrm{amp}\right)$ was measured with a galvanometer connected between the chamber and tube anode. One set of measurements for currents of about $10^{-10} \mathrm{amp}$ was made with a capacitance compensator and electrometer. ${ }^{11}$

Ionization chambers (fig. 1) of the following descriptions were used: A, cylinder, brass tube $7.2 \mathrm{~mm}$ inside diameter, cut away for about half its circumference and covered with $0.01 \mathrm{~mm}$ of $\mathrm{Al}$; collector rod $5 \mathrm{~mm}$ diameter; opening length $5 \mathrm{~cm}$. B, same as for A, except collector rod was $2 \mathrm{~mm}$ diameter. C, parallel plate, guarded-field ionization chamber ${ }^{12}$ with snout and back removed. $D$, parallel copper mesh (32), $1 \mathrm{~cm}$ spacing, $5 \mathrm{~cm}$ diameter. E, cylinder copper mesh (32), $8 \mathrm{~mm}$ inside diameter, collector $2 \mathrm{~mm}$ diameter. Chamber A was used with three different Lenard ray qualities, distinguished by $A_{1}, A_{2}$, and $A_{3}$ in the text and plots.

The Lenard ray beam was limited in cross section by a thin metal diaphragm placed in front of the tube window. This diaphragm had a diameter of $14 \mathrm{~mm}$, with $\mathrm{A}, \mathrm{B}$, and $\mathrm{E}, 2.9 \mathrm{~mm}$ with $\mathrm{C}$, and 1.9 $\mathrm{mm}$ with $\mathrm{D}$.

\section{EXPERIMENTAL RESULTS}

\section{CURRENT-VOLTAGE CURVES}

Curves for the ionization current as a function of the applied voltage are given in figure 2 for chambers A to D. Applied voltage rather than field strength is used as abscissas since the latter is indeterminate. It is seen in the case of curves $A_{1}, A_{2}$ and $A_{3}$, that noticeable collision ionization begins at about 1,000 volts, which corresponds roughly to a field strength of about $9 \mathrm{kv} / \mathrm{cm}$. In all other cases, this high field strength was not approached, and no evidence of collision ionization was noted.

It is also to be noted that at high field strengths, saturation is nearly reached, whereas for the very low fields used in parallel-plate chamber $\mathrm{C}$ there is no apparent evidence of approach to saturation.

It was not possible to vary the degree of the ionization by a known amount without at the same time introducing other variables, such as electron diffusion and change in velocity distribution. Hence, there is no simple direct way to ascertain whether or not the ionization current below saturation is proportional to the radiation intensity. This was attempted by indirect means. Previous studies have indicated the reliability of a faraday collector for measuring the Lenard current. ${ }^{2}$ The intensity of the beam was therefore varied by filtering with thin aluminum foil, and absorption curves of the resultant radiation obtained first with the faraday chamber and then with ionization chamber A at 900 volts, which is probably within 10 percent of saturation. This was done for three different distances, $d$, between the tube window and faraday collector or ionization chamber.

Figure 3 shows the results, where curves $M$ are for the ionization chamber and curves $\mathrm{N}$ are for the ratio $R$ of the ionization current to the faraday current. On the assumption that the measurements by

11 L. S. Taylor, BS J. Research 6, 807 (1931) RP306.

12 L. S. Taylor and G. Singer, BS J. Research 5, 507 (1930) RP211. 
the faraday chamber are correct, the relative sensitivity of the ionization chamber changes rapidly, increasing as the ionization becomes weaker.

An explanation for the apparently great disparity between the measurements by the two methods probably lies in the fact that whereas the faraday collector measures the total Lenard current, the ionization current gives only a measure of the fraction of the energy of the electrons absorbed within the ionization chamber. With no filter, fast cathode rays with low ionizing power pass completely

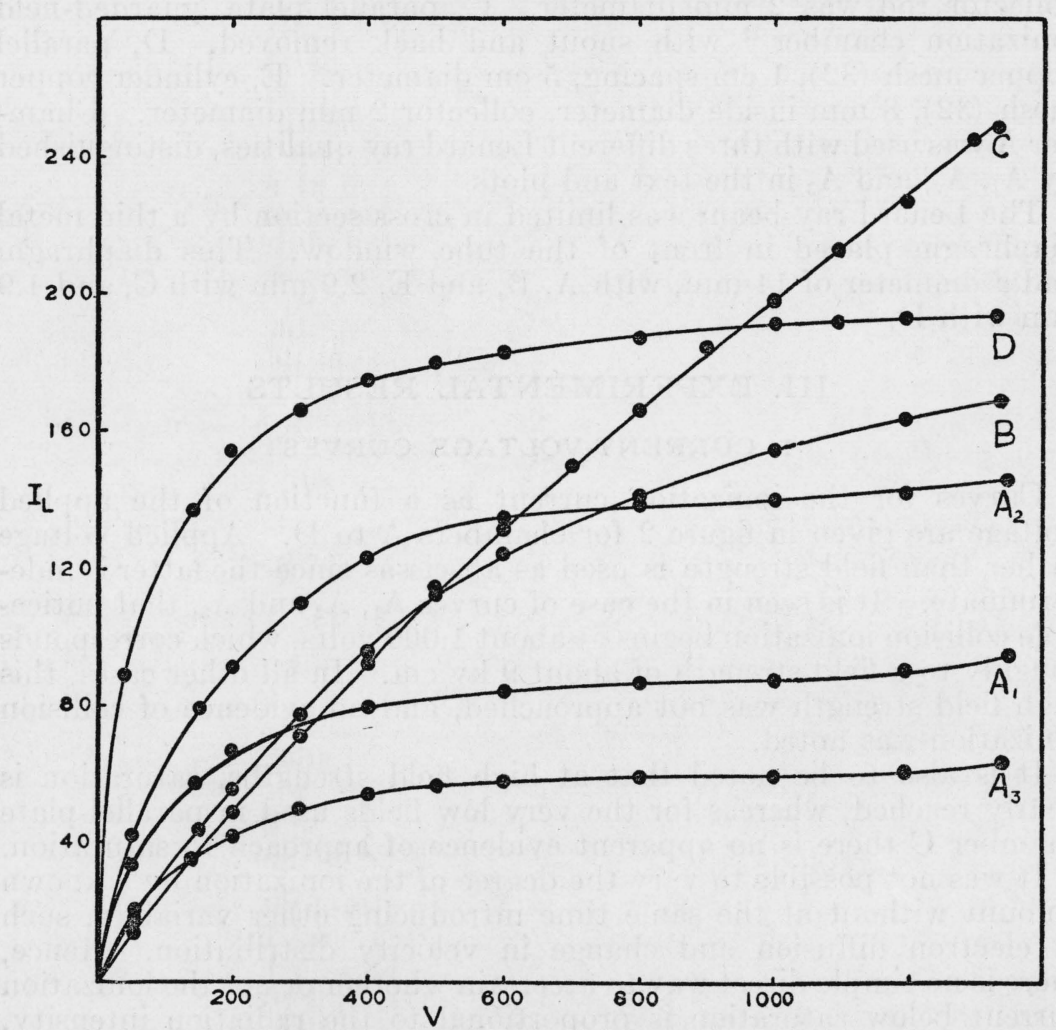

Figure 2.-Current-voltage curves for Lenard rays.

through the chamber. With increase in filtration an increasing fraction of the electrons terminate their paths within the chamber. and thereby produce a greater amount of ionization in accordance with Bragg's findings in terminal ionization.

\section{RECIPROCAL CURRENT-VOLTAGE CURVES}

Suggested by the results of plotting the reciprocal current against reciprocal voltage in the case of liquids (Mohler and Taylor) and highpressure gases (Zanstra), the above data were similarly treated on purely empirical grounds. ${ }^{13}$ Such a plot compresses the scale at the upper voltages so that an extrapolation to infinite fields is rendered practical.

13 L. S. Taylor, Phys. Rev. 48, 970 (1935). 
The data of figure 2 were accordingly plotted with the reciprocal current against the reciprocal voltage in figures 4 and 5 . It is seen that even down to comparatively low field strengths there is a linear relationship between the two quantities. In the case of chamber A, the field strength of $9.0 \mathrm{kv} / \mathrm{cm}$ gave very nearly the saturation current, as shown in figure 2. It would appear safe, therefore, to extrapolate the curve to $1 / V=0$, thereby deriving a magnitude for the ionization current $I_{\infty}$ at infinite field.

Similarly the other curves are extrapolated to $1 / V=0$, since they are straight lines even in the extreme case of chamber $\mathrm{C}$ where the maximum field strength was about $0.15 \mathrm{kv} / \mathrm{cm}$. Table 1 gives a summary of the experimental factors and also the ratio $I_{v} / I_{\infty}$ of the highest measured current, $I_{v}$, to the derived maximum $I_{\infty}$.

With the exception of chambers $\mathrm{C}$ and $\mathrm{D}$, the field strengths given do not have great significanceespecially those

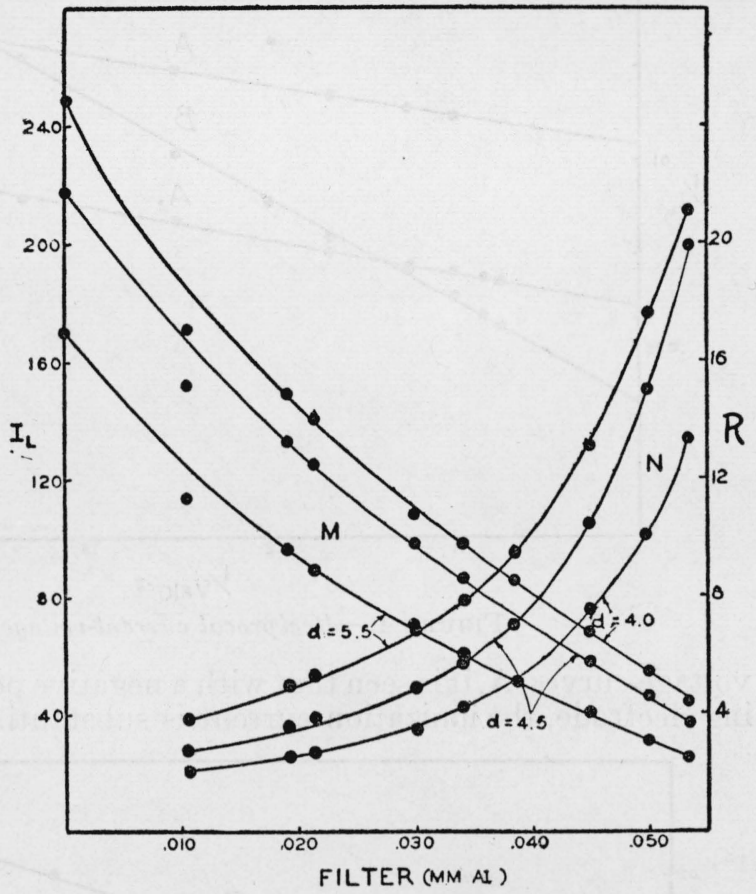

Figure 3.-Comparison of faraday chamber measurement and ionization measurement of Lenard ray.

marked with an asterisk-since in all cases, the field is radial and hence not uniform. In chambers $A_{1}, A_{2}$, and $A_{3}$ the radii of the inner and outer cylinders are 2.5 and $3.6 \mathrm{~mm}$, respectively, so the field is considerably more uniform than in chambers B and E.

TABLE 1.-Lenard ray intensities.

\begin{tabular}{|c|c|c|c|c|c|c|}
\hline Chamber & $\begin{array}{c}\text { Tube } \\
\text { distance } 1\end{array}$ & $\begin{array}{c}\text { Tube } \\
\text { diameter }\end{array}$ & Filter & $\begin{array}{l}\text { Maxi- } \\
\text { mum } \\
\text { field } \\
\text { strength }\end{array}$ & $I_{\infty}$ & $\frac{I_{0}}{I_{\infty}}$ \\
\hline $\begin{array}{l}A_{1} \\
A_{2} \\
A_{3}\end{array}$ & $\begin{array}{c}\text { Centi- } \\
\text { meter } \\
7.3 \\
4.6 \\
4.6 \\
4.6\end{array}$ & $\begin{array}{c}\text { Milli- } \\
\text { meter } \\
14 \\
14 \\
14 \\
14\end{array}$ & $\begin{array}{c}\begin{array}{c}\text { Milli- } \\
\text { meter }\end{array} \\
0.2 \mathrm{Al}\end{array}$ & $\begin{array}{r}\mathrm{kv} / \mathrm{cm} \\
9.1 \\
9.1 \\
9.1 \\
.3 .2\end{array}$ & $\begin{array}{c}a m p \times 10^{-6} \\
17.1 \\
53.8 \\
21.6 \\
91.2\end{array}$ & $\begin{array}{l}0.91 \\
.86 \\
.93 \\
.51\end{array}$ \\
\hline $\begin{array}{l}\mathrm{O} \\
\mathrm{D} \\
\mathrm{D} \\
\mathrm{E}_{1}-\mathrm{B}_{2-}\end{array}$ & $\begin{array}{l}6.6 \\
4.6 \\
4.6\end{array}$ & $\begin{array}{r}2.9 \\
1.9 \\
14\end{array}$ & -..... & $\begin{array}{l}.13 \\
.1 .4 \\
* 2.5 \\
\cdot 2.5\end{array}$ & $\begin{array}{l}29.3 \\
6.65 \\
77.8 \\
77.8\end{array}$ & $\begin{array}{l}.28 \\
.90 \\
.35 \\
.35\end{array}$ \\
\hline
\end{tabular}

1. Airpath between tube window and ionization chamber.
See explanatory text above. 
The effect of reversing the potential on the chamber is shown in figure 6 for the cylindrical chamber E. From the simple current-

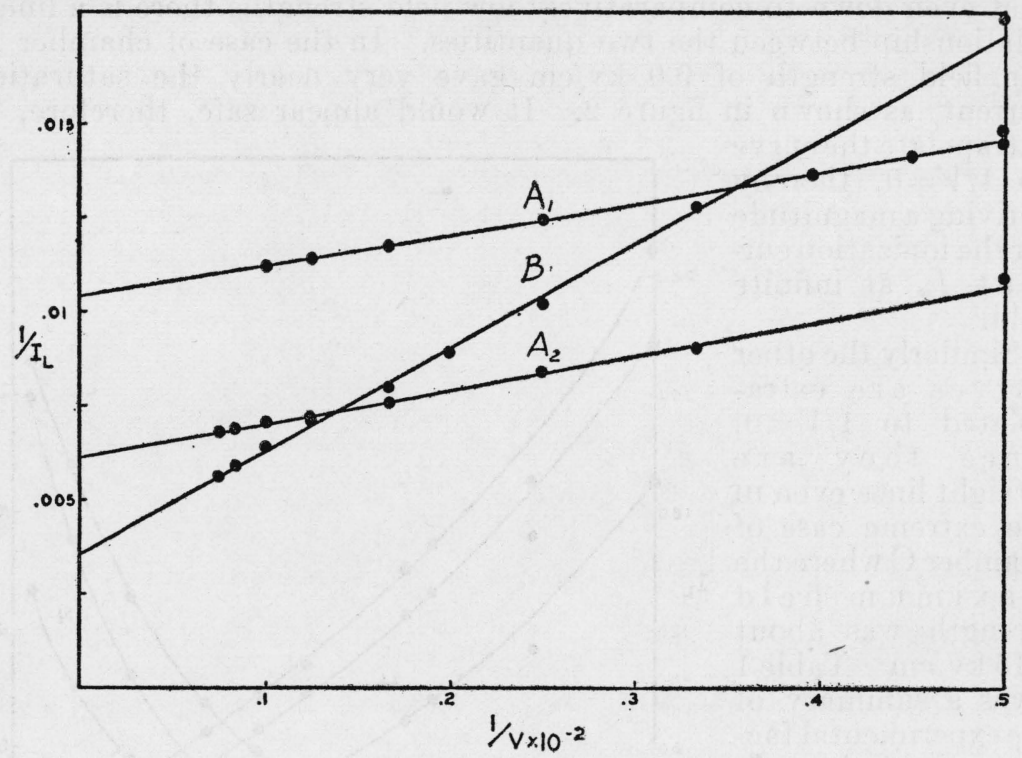

Figure 4.-Reciprocal current-voltage curves

voltage curves, $\mathrm{A}$, it is seen that with a negative potential on the collecting electrode, the ionization current is substantially larger than when

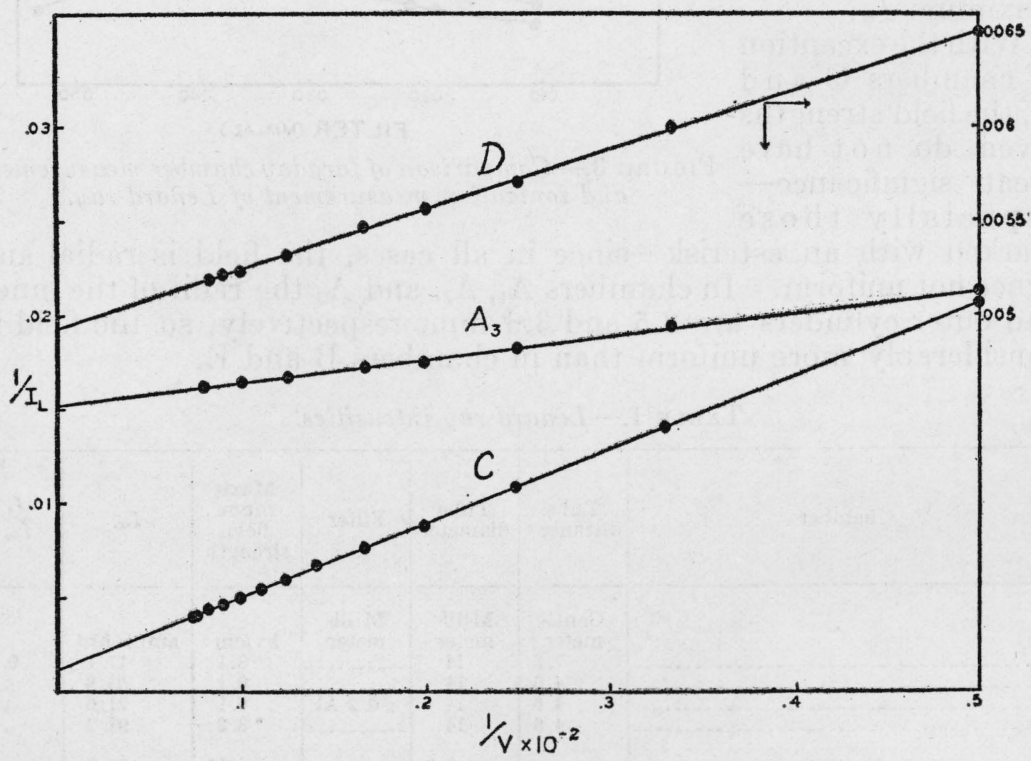

Figdre 5.-Reciprocal current-voltage curves

it is positive. Curves b show the same data plotted reciprocally; it is seen that although the points fall on two distinct straight lines, they extrapolate to the same value of $1 / I_{v}$ for $1 / V=0$. 
Included in table 1 are the values of $I_{\infty}$, which may be used for rough calculations of the ionization per unit volume, absolute values of which cannot be obtained for lack of sufficient definition of the beam and ionized volume. The effective volumes of chambers $\mathbf{A}_{\mathbf{2}}$ and $B$ are roughly in the ratio of $1: 2$, with which the respective values of $I_{\infty}$ agree.

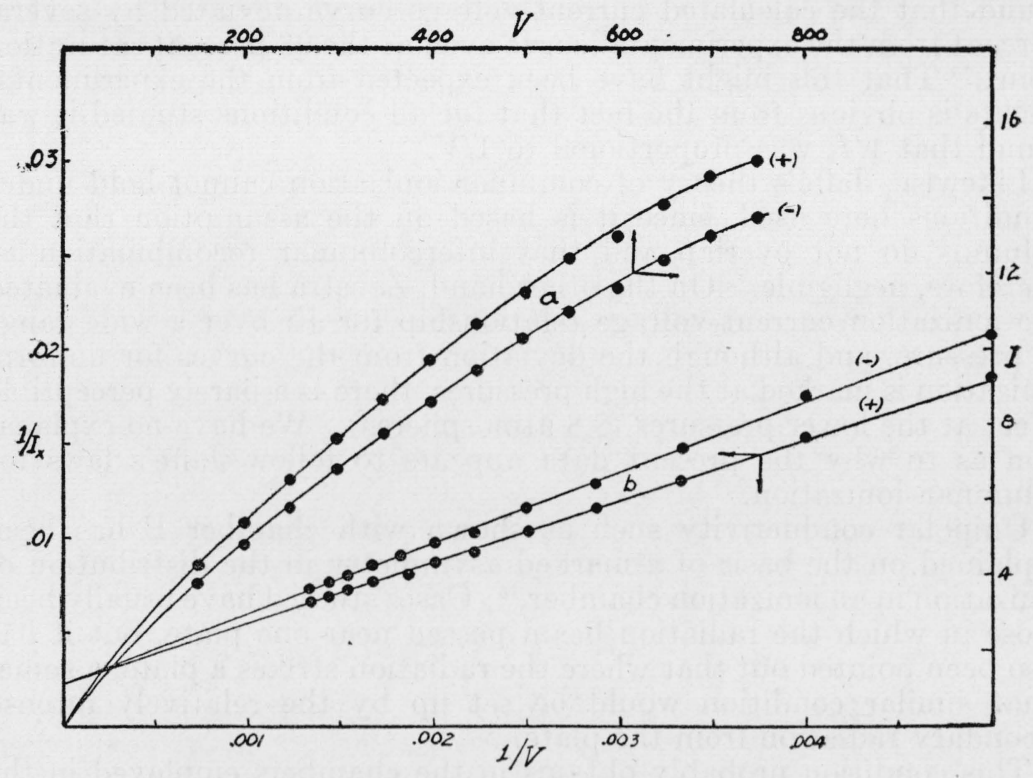

FiguRe 6.-Reciprocal current-voltage curves.

\section{DISCUSSION}

An attempt was made to explain the form of these ionization curves on the basis of some existing theory, but this is rendered too complicated by the inhomogeneous electric field in all chambers but $\mathrm{C}$ and $\mathrm{D}$. The relationship between ionization current and field strength for a uniformly ionized chamber has been found by others to agree fairly well with the theories of Thomson, ${ }^{14}$ Nice, and Seemann for X-rays and $\beta$ rays of moderate intensity (up to 5 roentgens per minute, for $\mathrm{X}$-rays). However, the intensities involved here are of the order of 1,000 times as large, and hence initial and intercolumnar recombination may be of greater importance here than in the cases of low ionization densities.

Thomson's equation for the ionization current $I_{x}$ at a given field strength $X$, where $I_{x}$ is small compared with $I_{\infty}$, is

$$
\frac{I^{2}{ }_{x}}{I_{\infty}-I_{x}}=\frac{\left(k_{1}+k_{2}\right)^{2} X^{2}}{\alpha / e . l}
$$

where $l$ is the plate separation, $\alpha$ the recombination coefficient, and $e$ the charge on the electron. Taking the square root of both sides of this equation it is seen (curve C, fig. 2) that for small values of $I_{x}$ there is a nearly linear relation between $I_{x}$ and $X$. The conditions in the equation may be approximately fulfilled by the chamber $\mathrm{C}$ and

14 See J. J. Thomson, Conduction of Electricity Through Gases (Cambridge, 1928). 
possibly $\mathrm{E}$ where the ratios $I_{x} / I_{\infty}$ were 0.28 and 0.35 , respectively. It is, indeed, true that the curves are linear for these chambers, but it is equally true that they are linear for the other chambers where $I_{x} / I_{\infty}$ is 0.9 , a magnitude too large for fulfilling the conditions of approximations. Assuming that this relation holds for the maximum ionization current measured with parallel-mesh chamber D, it was found that the calculated current-voltage curve deviated by several percent from the experimental curve even at the 98 percent saturation point. That this might have been expected from the experimental results is obvious from the fact that for all conditions studied it was found that $1 / I_{0}$ was proportional to $1 / V$.

Likewise, Jaffé's theory of columnar ionization cannot hold under conditions here used, since it is based on the assumption that the columns do not overlap and that intercolumnar recombination is, therefore, negligible. On the other hand, Zanstra has been evaluated the ionization current-voltage relationship for air over a wide range of pressure, and although the deviation from the curves for uniform ionization is marked at the high pressures, there is a barely perceptible effect at the lower pressures (8.8 atmospheres). We have no explanation as to why the present data appears to follow Jaffé's laws for columnar ionization.

Unipolar conductivity such as shown with chamber $\mathrm{E}$ has been explained on the basis of a marked asymmetry in the distribution of ionization in an ionization chamber. ${ }^{15}$ Cases studied have usually been those in which the radiation beam passed near one plate, but it has also been pointed out that where the radiation strikes a plate, a somewhat similar condition would be set up by the relatively intense secondary radiation from the plate.

This condition probably obtains in the chambers employed in the present work. In addition, since the Lenard rays are absorbed strongly by air, the ionization density will be substantially less at the emergent side than at the entrance side of the chamber. Of particular interest, however, is the fact that regardless of the sign of the potential on the plates, the relationship of $1 / I_{x}$ to $1 / X$ is linear at high field strengths. Moreover, under the same radiation conditions both curves extrapolate to identically the same value of $1 / I_{x}$ for $1 / X=0$. This latter fact would seem to definitely indicate the validity of a reciprocal current-voltage relationship and the permissibility of obtaining the true saturation current by extrapolation. For it is reasonable to expect the actual number of ions formed to be independent of any potential applied to the plates (except above the field strength where collision ionization occurs) and hence yield the same saturation current under conditions where no ions are lost by recombination.

The difference in ionization current at the lower fields might also be caused by electric field inhomogeneity, but it is interesting to note that exactly similar curves have been reported by Clay and Van Tijn ${ }^{16}$ for a parallel-plate chamber with air under high pressure and using gamma rays as the ionizing agent. We have also found similar results for parallel-plate air ionization chambers with very intense X-rays. ${ }^{17}$

WASHington, July 1, 1936.

15 E. Rutherford, Phil. Mag. [VI] 2, 210 (1901).

16 F. L. Mohler and L. S. Taylor. BS J. Research 13, 659 (1934) RP733.

17 Data to be published later. 